\title{
Active Shimmy Damping Using Direct Adaptive Fuzzy Control
}

\author{
Thai-Hoang Huynh, Gaétan Pouly, Jean-Philippe Lauffenburger, Michel Basset \\ University of Haute Alsace /MIPS Laboratory \\ ENSISA Lumière, 12, rue des frères Lumière - 68093 MULHOUSE Cedex, France
}

\begin{abstract}
The shimmy phenomenon is a self-excited limit cycle oscillation occurring in many physical rolling systems, particularly in aircraft nose landing gears (NLG). This paper presents a new active damping controller developed in the context of the European DRESS ("Distributed and Redundant Electro-mechanical nose gear Steering System") project for avoiding the shimmy oscillation. The controller based on the direct adaptive control approach, consists of two terms: the fuzzy adaptive term approximates the feedback linearization control law, and the stabilizing control term compensates the structural modelling error. The closed-loop system stability is proven by using Lyapunov theory. Simulation results corresponding to different test scenarios show that the proposed controller is able to effectively damp the shimmy phenomenon.
\end{abstract}

\section{INTRODUCTION}

The shimmy phenomenon is the self-excited oscillation of a wheel about its vertical steering axis, which may occur in many physical rolling systems such as aircraft nose wheels, automobiles, motorcycles... Shimmy is a violent and possibly dangerous vibration that can cause system malfunctions or even damages. In order to increase the understanding of this phenomenon on aircraft nose landing gears (NLG), researchers have elaborated different shimmy models. The developed shimmy models are mainly based on different ways of modelling the elasticity of tires, which plays a fundamental role in this dynamics (Stépán, 1991; Somieski, 1997; Sura and Suryanarayan, 2004). These models allow to analyse the stability and the response of shimmy oscillation, and to synthesize shimmy dampers. A classical solution to avoid shimmy is to increase the stiffness of the NLG by changing its material, and to increase the damping constant by using, for instance, additional passive dampers. One of the main drawbacks of passive damping solutions is that the damping characteristics may vary under changing load conditions or ground-tire interfaces. Recently, active shimmy damping approaches have been researched, and solutions have been proposed. Basically, active shimmy damping solutions rely on the use of sensors measuring the NLG behaviours, and a feedback control algorithm to calculate damping moments generated by an anti-shimmy actuator. Considering the control theory point of view, active damping solutions would increase the system performances because damping moments are generated based on feedback measurement signals corresponding to real operating conditions.

Different control approaches have been employed in active damping solutions, depending on the models of the oscillatory phenomena. If the oscillatory system can be described by a linear second order model, simple methods such as velocity feedback control, PD control, or linear filter could be adopted to adjust the damped ratio of the system (Houlston, et al., 2007; Høgsberg and Krenk, 2006). Modern control theories such as optimal control, adaptive control, robust control, fuzzy control or neural networks have recently been used to design damping controllers for more complex oscillatory systems (Choi and Han, 2003; Kawabe et al., 2006).

Although many works are related to active damping, less works concern active shimmy damping. In (Brewer and Skele, 1975), a feedback signal proportional to the angular velocity of the NLG wheel is used to control the hydraulic actuator pressure. Switching control method is applied to stabilize the NLG dynamics in (Zefran and Burdick, 1998). Goodwine and Stépán (2000) suggested a control algorithm for a simple nonlinear model of the NLG based on the feedback linearization law. One drawback of the above solutions is that the control designs are based on a nominal shimmy model. For that reason, the control performances may change if the system parameters are time-varying, e.g. the vertical load or the tire dynamics.

Recently, adaptive control of nonlinear systems using neural networks and fuzzy systems has been strongly developed, not only in theory, but also in applications. This control approach is able to cope with uncertainties and time-varying dynamics, so it is a potential solution to the active shimmy damping problem. With this motivation, in the framework of the FP7 supported European DRESS project, an adaptive controller is developed to actively damp the shimmy phenomenon in aircraft NLGs. In fact, the aim of the DRESS project is to investigate not only new active shimmy damping solutions, but also new NLG steering system using electro-mechanical actuators to improve the competitiveness and aircraft safety. In this paper, a direct adaptive fuzzy active damping solution is discussed. The rest of this paper is organized as follows. In section 2, a NLG model is developed for control design. Section 3 briefly summarizes a direct adaptive fuzzy control algorithm. Section 4 presents the design of the active damping controller and the simulation results corresponding to different test scenarios. Finally, section 5 concludes this paper. 


\section{THE DYNAMICS OF THE SHIMMY PHENOMENON}

In this paper, the simplified NLG model presented in (Somieski, 1997) is further developed for active damping design by integrating an actuator. The considered system consists of the mechanical dynamics of the actuator, the torsional dynamics of the NLG, and the forces and moments describing the tire's elasticity. The diagram of this model is illustrated in figure 1.

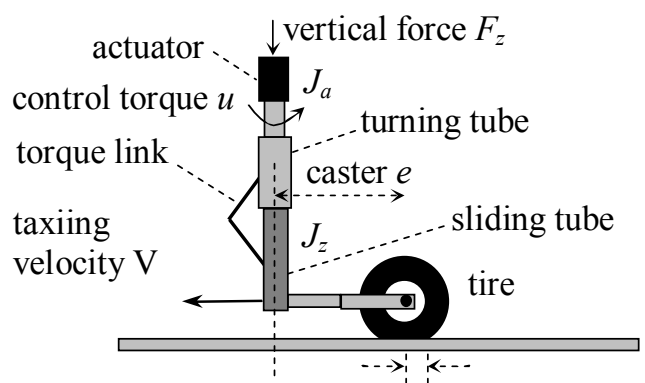

swivel axis half contact length $a$

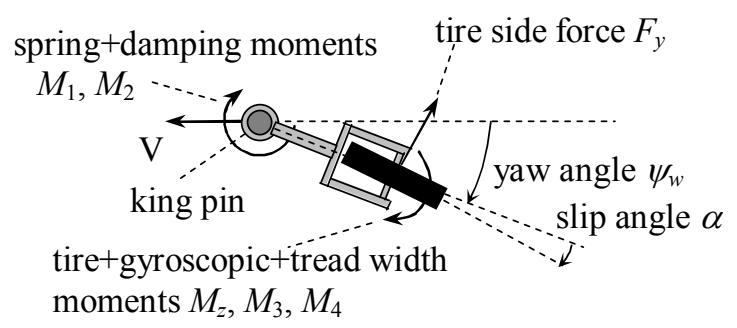

Fig. 1. Nose landing gear model

\subsection{Nonlinear mathematic model}

The input to the model is the control torque $u$ provided by an actuator, and the output of the model is the angle $\psi_{w}$ of the wheel about its vertical rotating axis. Suppose that the link between the actuator and the turning tube is rigid, this means that the angle of the actuator output $\psi_{a}$ is equal to the angle of the turning tube. Applying Newton's second law to the rotating movements of the actuator and the NLG leads to the following equations:

$$
\begin{aligned}
& J_{a} \ddot{\psi}_{a}=u-B_{a} \dot{\psi}_{a}-M_{1}-M_{2} \\
& J_{z} \ddot{\psi}_{w}=M_{1}+M_{2}+M_{3}+M_{4}
\end{aligned}
$$

where $J_{a}$ and $J_{z}$ are the moments of inertia of the actuator and of the NLG, $B_{a}$ is the viscous friction constant of the actuator, $M_{1}=c\left(\psi_{a}-\psi_{w}\right)$ is the torsional moment provided by the torque link, $M_{2}=k\left(\dot{\psi}_{a}-\dot{\psi}_{w}\right)$ is the damping moment from viscous friction in the bearings of the oil-pneumatic shock absorber, $M_{3}$ is the tire moment caused by the lateral tire deformations due to side slip, and $M_{4}$ is the tire damping moment related to the yaw rate. The following equations summarize the nonlinear characteristics of the tire, which are discussed in detailed in (Somieski, 1997).

$M_{3}=M_{z}-e F_{y}$

$$
\begin{aligned}
& F_{y}= \begin{cases}c_{F \alpha} \alpha F_{z} & \text { for }|\alpha| \leq \delta \\
c_{F \alpha} \delta F_{z} \operatorname{sign}(\alpha) & \text { for }|\alpha|>\delta\end{cases} \\
& M_{z}= \begin{cases}c_{M \alpha} F_{z} \frac{\alpha_{g}}{180} \sin \left(\frac{180}{\alpha_{g}} \alpha\right) & \text { for }|\alpha| \leq \alpha_{g} \\
0 & \text { for }|\alpha|>\alpha_{g}\end{cases} \\
& M_{4}=\frac{\kappa}{v} \dot{\psi}_{w} \\
& \dot{y}_{l}+\frac{v}{\sigma} y_{l}=v \psi_{w}+(e-a) \dot{\psi}_{w} \\
& \alpha \approx \arctan \alpha=\frac{y_{l}}{\sigma}
\end{aligned}
$$

where $M_{z}$ is the self-aligning torque, $F_{y}$ is the side force, $F_{z}$ is the vertical force, $v$ is the aircraft ground speed, $y_{l}$ is the lateral displacement of the leading contact point of the tire, $\alpha$ is the slip angle of the wheel, $e$ is the caster length, $a$ is half of the contact length, and $c_{F \alpha}, c_{F \alpha}, \kappa, \delta, \alpha, \sigma$ are constants as defined in (Somieski, 1997).

It is important to note that there are two nonlinearities in the model related to the elasticity of the tires. These nonlinearities may cause a limit cycle in the system. For this reason, the NLG is rather difficult to control.

\subsection{State space representation}

To design the adaptive damping controller, the state space representation of the NLG model is needed. By choosing the state variables $x_{1}=\psi_{w}, \quad x_{2}=\dot{\psi}_{w}, \quad x_{3}=y_{l}, \quad x_{4}=\psi_{a}$, $x_{5}=\dot{\psi}_{a}$, the nonlinear dynamics presented above can be expressed as:

$$
\left\{\begin{array}{l}
\dot{x}_{1}=x_{2} \\
\dot{x}_{2}=\frac{c\left(x_{4}-x_{1}\right)}{J_{z}}+\frac{k\left(x_{5}-x_{2}\right)}{J_{z}}+f_{1}\left(x_{3}\right)+f_{2}\left(x_{2}\right) \\
\dot{x}_{3}=v x_{1}+(e-a) x_{2}-\frac{v}{\sigma} x_{3} \\
\dot{x}_{4}=x_{5} \\
\dot{x}_{5}=-\frac{B_{a} x_{5}}{J_{a}}-\frac{c\left(x_{4}-x_{1}\right)}{J_{a}}-\frac{k\left(x_{5}-x_{2}\right)}{J_{a}}+\frac{1}{J_{a}} u
\end{array}\right.
$$

where:

$$
\begin{aligned}
& f_{1}\left(x_{3}\right)=\frac{M_{3}(\alpha)}{J_{z}}=\frac{M_{3}\left(y_{l} / \sigma\right)}{J_{z}} \\
& f_{2}\left(x_{2}\right)=\frac{M_{4}\left(\dot{\psi}_{w} / v\right)}{J_{z}}
\end{aligned}
$$

The output of the system is $y=\psi_{w}=x_{1}$. Consecutively taking the derivatives of the output leads to Equ. (12):

$\dddot{y}=\frac{c\left(\dot{x}_{4}-\dot{x}_{1}\right)}{J_{z}}+\frac{k\left(\dot{x}_{5}-\dot{x}_{2}\right)}{J_{z}}+\dot{f}_{1}\left(x_{3}\right) \dot{x}_{3}+\dot{f}_{2}\left(x_{2}\right) \dot{x}_{2}$

Substituting the derivatives of the state variables in (9) into (12), it is obvious that the input $u$ appears on right hand side 
of the result. This means that the system has the relative degree of 3 , and can be described by the following equation:

$$
\dddot{y}=a(x)+b(x) \cdot u
$$

where $x=\left[x_{1}, x_{2}, \ldots, x_{5}\right]^{T}$ is the system's state vector, $a(x)$ and $b(x)$ are nonlinear functions. The explicit descriptions of the two nonlinear functions can be obtained after some mathematical manipulations. However, even if the exact expressions are calculated, they might not well describe the dynamics of the system when it is operating, because of timevarying parameters such as vertical force or tire characteristics. For that reason, $a(x)$ and $b(x)$ are considered as unknown functions, and adaptive control theory is adopted to cope with this uncertainty.

\section{DIRECT ADAPTIVE FUZZY CONTROL}

\subsection{Control strategy}

Consider the class of SISO nonlinear systems described by the following state equation:

$$
\left\{\begin{array}{l}
\dot{x}=f(x)+g(x) u \\
y=h(x)
\end{array}\right.
$$

where $x=\left[x_{1}, x_{2}, \ldots, x_{n}\right]^{T} \in \mathfrak{R}^{n}, u \in \Re, y \in \mathfrak{R}$ are respectively the system states, input and output; $f(x) \in \mathfrak{R}^{n}, g(x) \in \mathfrak{R}^{n}, h(x) \in \mathfrak{R}$ are smooth functions describing the dynamic of the system. If the system has the relative degree of $r(r \leq n)$, then its output can be expressed as (Sastry and Bodson, 1989):

$$
y^{(r)}=a(x)+b(x) u
$$

where $a(x)=L_{f}^{r} h(x)$ and $b(x)=L_{g} L_{f}^{r-1} h(x) \neq 0$. The notation $L_{f} h(x)$ is the Lie derivative of the function $h(x)$ with respect to $f(x)$.

The objective is to design a feedback control law to drive the system output $y$ tracking a reference output $y_{m}$. With the assumptions that all the states of the system are measurable and available for feedback, and the reference output $y_{m}(t)$ and its derivatives up to the $r$ th order are measurable and bounded, the mentioned control objective can be met by applying the feedback linearization control law (Spooner and Passino, 1996):

$$
u^{*}(t)=\frac{1}{b(x)}[-a(x)+v(t)]
$$

where $v(t)=y_{m}^{(r)}+\bar{e}_{s}+\eta e_{s}$ is the pseudo input to the linearized system, $e_{s}=e_{o}^{(r-1)}+k_{1} e_{o}^{(r-2)}+\ldots+k_{r-1} e_{o}$ is the tracking error, $e_{o}=y_{m}-y$ is the output error, and $\bar{e}_{s}=\dot{e}_{s}-e_{o}^{(r)}$. The coefficients $k_{i}(i=1, \ldots, r-1)$ are chosen so that the polynomial $\Delta(s)=s^{(r-1)}+\quad+k_{1} s^{(r-2)}+\ldots+k_{r-2} s+k_{r-1}$ is Hurwitz. It is not difficult to prove that the closed-loop system with the control law (16) is stable and the output error asymptotically approaches zero.
Assume that the functions $a(x)$ and $b(x)$ describing the system dynamics are unknown; the ideal control law cannot be implemented. Instead, the control law (16) is approximated by a universal approximator of the following form:

$$
\hat{u}(x, \theta)=\theta^{T} \xi(x)
$$

where $\xi(x)$ is the basic function vector and $\theta$ is the parameter vector. In principle, any type of universal approximator can be used to implement (17). In this work, however, a fuzzy model is employed because it is possible to integrate human knowledge to define the basic function vector by choosing the membership functions. The fuzzy model consists of fuzzy rules of the following form:

If $x_{1}$ is $F_{i 1}$ and $\ldots$ and $x_{n}$ is $F_{i n}$ then $\hat{u}=\theta_{i}$

where $F_{i j}$ is the fuzzy set of the state variable $j$ used in rule $i$ $(i=1 . . n, j=1 . . m)$. Using product norm to implement and operation, and weighted average method for defuzzification, the output of the fuzzy system (18) can be expressed in the form (17) with $\theta=\left[\theta_{1}, \ldots, \theta_{m}\right]^{T}, \xi(x)=\left[\xi_{1}(x), \ldots, \xi_{m}(x)\right]^{T}$, and:

$$
\xi_{i}(x)=\frac{\prod_{j=1}^{n} \mu_{i j}\left(x_{j}\right)}{\sum_{i=1}^{m} \prod_{j=1}^{n} \mu_{i j}\left(x_{j}\right)}, \quad(i=1 . . n)
$$

where $\mu_{i j}\left(x_{j}\right)$ is the membership function of the fuzzy set $F_{i j}$. The parameter vector $\theta$ is updated online so that the approximation error between $\hat{u}$ and $u^{*}$ is minimal. Define the optimal parameter vector as:

$$
\theta^{*}=\arg \min _{\theta}\left\{\sup _{x}\left|\theta^{T} \xi(x)-u^{*}\right|\right\}
$$

It is proven that the fuzzy system (18) can approximate smooth nonlinear functions with arbitrary small error if the number of fuzzy rules is large enough (Kosko, 1994). In general cases, $\hat{u}$ is not identical to $u^{*}$ even when $\theta \rightarrow \theta^{*}$. Let $\delta_{u}(x)$ the structure error, the ideal control law can be expressed:

$$
u^{*}(x)=\theta^{* T} \xi(x)+\delta_{u}(x)
$$

The difference between the identified control and the ideal one is:

$$
\hat{u}(x)-u^{*}(x)=\widetilde{\theta}^{T} \xi(x)-\delta_{u}(x)
$$

where $\widetilde{\theta}=\theta-\theta^{*}$ is the parameter error.

Because of the structure error, an additional stabilizing control term $u_{s}$ is used to compensate the modelling error and to make sure that the closed-loop system is stable. As a result, the final control law is of the form:

$$
u=\hat{u}+u_{s}
$$

The block diagram of the controller is illustrated in figure 2 . The parameter update law and the stabilizing term are discussed in detailed in the stability analysis below. 


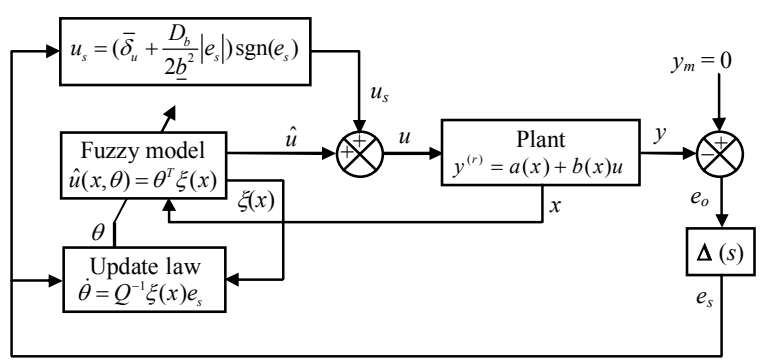

Fig. 2. Direct adaptive fuzzy controller.

\subsection{Stability analysis}

To prove the stability of the close-loop system, the following assumptions are required:

Assumption 1: $b(x)$ is finite and bounded away from zero, and its sign is unchanged. For simplicity the proof below only considers the case $b(x)>0$, but we have similar result when $b(x)<0$. Assume that $b(x)$ satisfies $0<\underline{b} \leq b(x) \leq \bar{b}<\infty$.

Assumption 2: The derivative of $b(x)$ is bounded, meaning that it exists a positive constant $D_{b}$ so that $|\dot{b}(x)| \leq D_{b}$.

Assumption 3: The structure error is bounded, meaning that there is a constant $\bar{\delta}_{u}$ so that $\left|\delta_{u}(x)\right| \leq \bar{\delta}_{u}$.

The $r^{\text {th }}$ derivative of the output error is:

$$
\begin{aligned}
e_{o}^{(r)} & =y_{m}^{(r)}-y^{(r)}=y_{m}^{(r)}-(a+b u) \\
& =y_{m}^{(r)}-v-b\left(u-u^{*}\right)=-\bar{e}_{s}-\eta e_{s}-b\left(\hat{u}+u_{s}-u^{*}\right) \\
& =-\bar{e}_{s}-\eta e_{s}-b \widetilde{\theta}^{T} \xi+b \delta_{u}-b u_{s}
\end{aligned}
$$

Substituting $\bar{e}_{s}$ into (24) leads to:

$$
\dot{e}_{s}+\eta e_{s}=-b \widetilde{\theta}^{T} \xi+b \delta_{u}-b u_{s}
$$

Consider the Lyapunov candidate function:

$$
V=\frac{1}{2 b} e_{s}^{2}+\frac{1}{2} \widetilde{\theta}^{T} Q \widetilde{\theta}
$$

where $Q \in \Re^{d \times d}(d=\operatorname{dim} \theta)$ is a positive definite matrix. Take the derivative of $V$ with respect to time and notice that $\dot{\tilde{\theta}}=\dot{\theta}$, we have:

$$
\begin{aligned}
\dot{V} & =\frac{1}{b} e_{s} \dot{e}_{s}-\frac{\dot{b}}{2 b^{2}} e_{s}^{2}+\widetilde{\theta}^{T} Q \dot{\theta} \\
& =\frac{e_{s}}{b}\left(-\eta e_{s}-b \widetilde{\theta}^{T} \xi+b \delta_{u}-b u_{s}\right)-\frac{\dot{b}}{2 b^{2}} e_{s}^{2}+\dot{\widetilde{\theta}}^{T} Q \dot{\theta} \\
& =-\frac{\eta e_{s}^{2}}{b}-e_{s} u_{s}+e_{s} \delta_{u}+\dot{\widetilde{\theta}}\left(Q \dot{\theta}-\xi e_{s}\right)-\frac{\dot{b}}{2 b^{2}} e_{s}^{2}
\end{aligned}
$$

Chose the parameter update law to cancel the parameter error as follow:

$$
\dot{\theta}=Q^{-1} \xi_{e_{s}}
$$

Substitute the update law (28) into (27), we have:

$$
\begin{aligned}
\dot{V} & =-\frac{\eta e_{s}^{2}}{b}-e_{s} u_{s}+e_{s} \delta_{u}-\frac{\dot{b}}{2 b^{2}} e_{s}^{2} \\
& \leq-\frac{\eta e_{s}^{2}}{\bar{b}}-u_{s} e_{s}+\left|e_{s}\right|\left(\bar{\delta}_{u}+\frac{D_{b}}{2 \underline{b}^{2}}\left|e_{s}\right|\right)
\end{aligned}
$$

Chose the stabilizing control as follow:

$$
u_{s}=\left(\bar{\delta}_{u}+\frac{D_{b}}{2 \underline{b}^{2}}\left|e_{s}\right|\right) \operatorname{sgn}\left(e_{s}\right)
$$

Substitute (30) into (29), and notice that $e_{s} \operatorname{sgn}\left(e_{s}\right)=\left|e_{s}\right|$, we have:

$$
\dot{V} \leq-\frac{\eta e_{s}^{2}}{\bar{b}} \leq 0
$$

Since $V$ is a quadratic function and $\dot{V} \leq 0$, the control system is proven to be stable. It is clear that $V \in \mathcal{L}_{\infty}$, which implies $e_{s} \in \mathcal{L}_{\infty}$ and $\tilde{\theta} \in \mathcal{L}_{\infty}$. Because of the assumptions 1-3 and $e_{s} \in \mathcal{L}_{\infty}$, from (30) we infer $u_{s} \in \mathcal{L}_{\infty}$, and consequently, from (25) we have $\dot{e}_{s} \in \mathcal{L}_{\infty}$. From the definition of $e_{s}$, we have $e_{o}^{(j)}=G_{j}(s) e_{s}$, where $G_{j}(s)=s^{j} / \Delta(s)(j=1, \ldots, r-1)$, and $G_{j}(s)$ is stable because $\Delta(s)$ is Hurwitz, so $e_{o}^{(j)} \in \mathcal{L}_{\infty}(j=1, \ldots, r-1)$. This means the output error and its derivatives up to $(r-1)^{\text {th }}$ order are bounded. From (31) we can infer:

$$
\int_{0}^{\infty} \frac{\eta e_{s}^{2}}{\bar{b}} d t \leq-\int_{0}^{\infty} \dot{V} d t=V(0)-V(\infty)<\infty
$$

which implies that $e_{s} \in \mathcal{L}_{2}$. Because $e_{s} \in \mathcal{L}_{2} \cap \mathcal{L}_{\infty}$ and $\dot{e}_{s} \in \mathcal{L}_{\infty}$, by Barbalat's lemma (Satry and Bodson, 1989) we conclude that $\lim _{t \rightarrow \infty} e_{s}(t)=0$, and consequently $\lim _{t \rightarrow \infty} e_{o}(t)=0$. This means that the system output asymptotically approaches to the desired output.

\section{ACTIVE SHIMMY DAMPING DESIGN AND RESULTS}

\subsection{Controller design}

The active shimmy damping controller for an aircraft NLG is designed on the direct adaptive fuzzy control algorithm discussed in the previous section. Suppose that the system moves straight forward, then the reference output $y_{m}$ can be set to zero for the problem of shimmy damping. The following section details the design of the active damping controller corresponding to the NLG parameters given in (Somieski, 1997), and the actuator parameters chosen as $J_{a}=$ $0.1 \mathrm{~kg} \cdot \mathrm{m}^{2}$, and $B_{a}=0.1 \mathrm{~N} \cdot \mathrm{m} / \mathrm{rad} / \mathrm{s}$.

Tracking error. As presented in section 2, the considered NLG model is a $5^{\text {th }}$ order nonlinear system with the relative degree of 3 . The tracking error is $e_{s}(t)=\ddot{e}_{o}+k_{1} \dot{e}_{o}+k_{2} e_{o}$, with $k_{1}=100, k_{2}=25$.

Fuzzy system. Three Gaussian membership functions for state variable $x_{1}$, and two membership functions for each other state variables have been designed. The membership 
functions are uniformly distributed in the range $\left(-\bar{x}_{i}, \bar{x}_{i}\right)$ for each state variable, with $\bar{x}_{1}=\pi / 10, \bar{x}_{2}=\pi, \bar{x}_{3}=0.1$, $\bar{x}_{4}=\pi / 10$, and $\bar{x}_{5}=\pi$. The fuzzy system consists of 48 rules of the form (18).

Stabilizing control term. From the mathematical description of the NLG discussed in section 2, it is easy to verify that $b(x)=k / J_{a} J_{z}$. Suppose that the uncertainty of the parameters is $10 \%$, the lower bound of $b(x)$ can be calculated as $\underline{b}=74.38$. In the ideal case: $\dot{b}(x)=0$, because $b(x)$ is constant for the considered plant. However, the plant's parameters may be slowly time-varying, so we can assume that $|\dot{b}(x)|$ is bounded by a small constant. In this design, we choose $D_{b}=1$. The last parameter needed to calculate $u_{s}$ is the bound $\bar{\delta}_{u}$ of the structure error. Because the fuzzy model defined above is flexible enough, $\bar{\delta}_{u}$ is chosen to be small to limit the chattering phenomenon, which may occur in the switching stabilizing control signal.

\subsection{Simulation results}

To illustrate the performance of the proposed shimmy damping controller, simulations have been done with three different test scenarios.

Scenario 1: Constant ground speed, pulse disturbance. In this simulation, the system is supposed to have a constant ground speed of $v=80 \mathrm{~m} / \mathrm{s}$, and the disturbance is a torque pulse of $1000 \mathrm{Nm}$ during 0.1 second acting directly on the vertical axis at the wheel level. If the damping constant of the NLG is low, e.g. $k=10 \mathrm{~N} \cdot \mathrm{m} / \mathrm{rad} / \mathrm{s}$, shimmy oscillation occurs. Figure 4 shows the shimmy oscillation considering that the turning tube is strictly kept at zero position; the oscillation is even more drastic in the case of free castoring. Figure 5 shows the response of the NLG with the active damping controller in action. It is obvious that no shimmy appears, and the oscillation is damped within two cycles. However, as the figure reveals, there is a small bias angle when the disturbance remains; the wheel angle only returns to its original position when the disturbance disappears. This behaviour of the proposed active damping controller is quite similar to what of current passive shimmy damping solutions. Notice that the main purpose of the designed controller is not to drive the wheel, but to avoid the shimmy oscillation. For that reason, the control algorithm just requires the maximum torque of about $500 \mathrm{Nm}$ to effectively prevent the oscillation, while the disturbance magnitude is $1000 \mathrm{~N} \cdot \mathrm{m}$. In fact, it is possible to choose the design parameters of the adaptive controller so that the wheel angle will return to its zero position even when the disturbance exists, but in this case the control torque must be larger than the disturbance.

Scenario 2: Constant ground speed, random disturbance. The purpose of this test is to investigate the effect of the shimmy active damping controller while the NLG is used at maximum speed, and considering the influence of the roughness of the runway on the tire. This aspect is modelled by a random disturbance, which is a white noise with zero

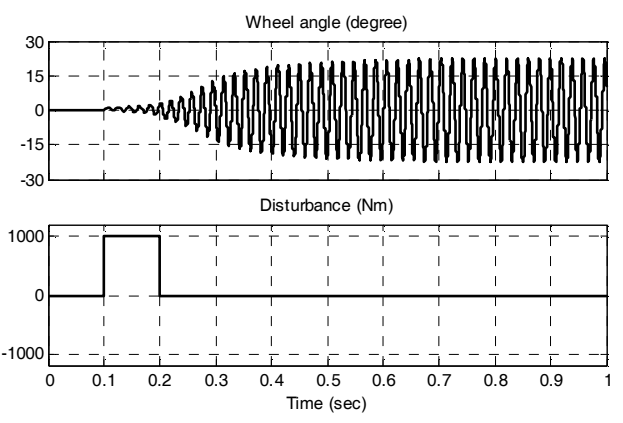

Fig. 4. Shimmy caused by a pulse disturbance
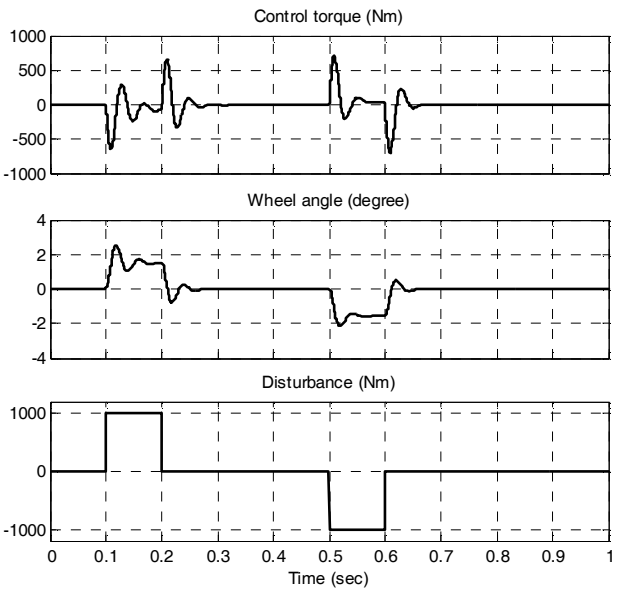

Fig. 5. Active shimmy damping result (scenario 1)

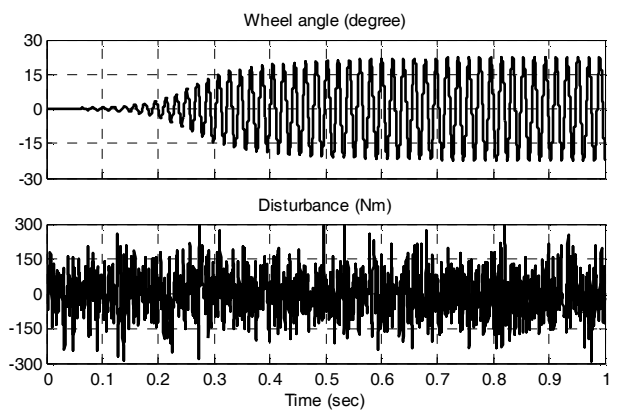

Fig. 6. Shimmy caused by random disturbances

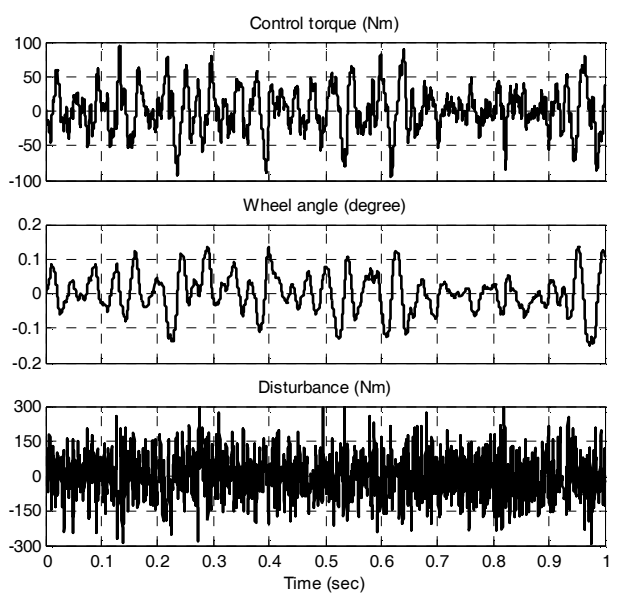

Fig. 7. Active shimmy damping result (scenario 2) 
mean and standard deviation of $100 \mathrm{~N} \cdot \mathrm{m}$. Without any shimmy damper, this disturbance causes shimmy oscillation (figure 6). With the proposed active damping controller, as shown in figure 7 , shimmy does not occur and the variation of the wheel angle is very small (less than 0.2 degrees). In practice, this small variation cannot cause any damage or malfunction to the NLG.

Scenario 3: Variant ground speed, random disturbance. This test is to investigate the behaviour of the NLG when the system is under varying speed conditions. This simulation has been performed with the forward velocity $v$ changing from 0 to $80 \mathrm{~m} / \mathrm{s}$ with an acceleration of $4.9 \mathrm{~m} / \mathrm{s}^{2}$. The disturbance is a white noise as discussed above. Figure 8 shows the simulation result with the active shimmy damping controller in action, there is no shimmy occurs. Notice that the variation of the wheel angle at low speed is larger than at high speed, meaning that the influence of the forward acceleration on the behaviour of the NLG is stronger at low speed. This is because the tire damping moment $M_{4}$ is more sensitive to the acceleration when the forward velocity is low.
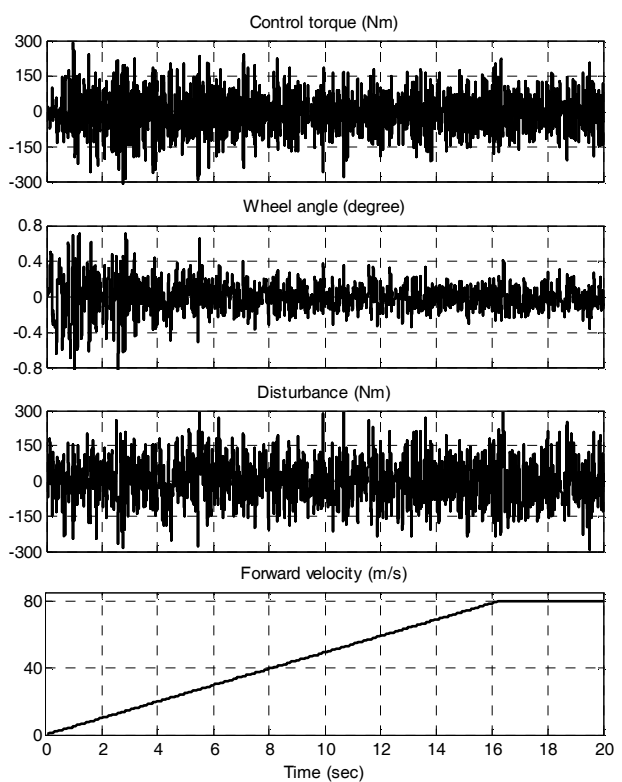

Fig. 8. Active shimmy damping result (scenario 3)

\section{CONCLUSIONS}

The paper showed a first attempt at designing an active shimmy damping controller based on direct adaptive fuzzy approach for an aircraft NLG. The proposed controller, which consists of an adaptive term and a stabilizing term, is designed based on Lyapunov stability theory. The adaptive term is implemented by a fuzzy system with tuneable parameters. Simulation results show that the designed controller can effectively avoid shimmy phenomenon in test scenarios with different disturbance forms and forward velocity profiles. The main drawback of the proposed active damping solution is that the control algorithm needs to feedback all the state variables. Nevertheless, this requirement might not be met in some practical cases. Shimmy active damping using output feedback adaptive control will be considered in the future.

\section{ACKNOWLEDGEMENT}

This work has been carried out in the context of the DRESS research program, funded by the European Union. The authors would like to thank Jean-Pierre Garcia, Stephane Dellac and Paul-Louis Levy from Messier Bugatti, coordinator of the DRESS project, for their interests and discussions.

\section{REFERENCES}

Choi, S.-B., and S.-S. Han (2003). Hळ control of electrorheological suspension system subjected to parameter uncertainties, Mechatronics, 13, pp. 639-657.

Brewer, H.K., and P. Skele (1975). Active shimmy control system, Technical Report AFFDL-TR-75-136, December 1975.

Goodwine, B., G. Stépán (2000). Controlling unstable rolling phenomena, Journal of Vibration and Control, 6, pp.137-158.

Høgsberg, J. R. and S. Krenk (2006). Linear control strategies for damping of flexible structures, Journal of Sound and Vibration, 293, pp. 59-77.

Houlston, P. R., S. D. Garvey, and A. A. Popov (2007). Modal control of vibration in rotating machines and other generally damped systems, Journal of Sound and Vibration, 302, pp.104-116.

Kawabe, H., N. Tsukiyama, K. Yoshida (2006), Active vibration damping based on neural network theory, Materials Science and Engineering A, 442, pp. 547-550.

Kosko, B. (1994). Fuzzy Systems as Universal Approximators, IEEE Transactions on Computers, 43(11), pp. 1329-1333.

Sastry, S. and M. Bodson (1989). Adaptive Control: Stability, Convergence, and Robustness, Englewood Cliffs, NJ, Prentice Hall.

Somieski, G. (1997). Shimmy Analysis of a Simple Aircraft Nose Landing Gear Model Using Different Mathematical Methods, Aerospace Science and Technology, 87, pp 545-555.

Stépán, G. (1991). Chaotic motion of wheel, Vehicle System Dynamics, 20, pp. 341 -351.

Spooner, J.T., and K.M. Passino (1996). Stable adaptive control using fuzzy systems and neural networks, IEEE Trans. Fuz. Syst, 4(3), pp.339-359.

Sura, N. K., and S. Suryanarayan (2004). Stability and Response Studies on Simplified Models of Nose-wheel Landing Gear with Hard Tires, Aerospace Engineering, 85, pp. 29-36.

Zefran, M., and J.W. Burdick (1998). Stabilization of systems with changing dynamics by means of switching, Proceedings of IEEE Int. Conf. on Robotics and Automation, 2, pp. 1090-1095. 